\title{
Effects of modified Shoutaiwai recipe on integrin $\beta 3$ and leukemia-inhibitory factor in endometrium of controlled ovarian hyperstimulation mice during the implantation window
}

\author{
X.Y. Chen ${ }^{1}$, J. Chen ${ }^{1}$, Z.Y. Wang ${ }^{2}$, X.H. Yu ${ }^{1}$, B.X. Wei ${ }^{1}$ and X.H. Wu ${ }^{1}$ \\ ${ }^{1}$ The Maternal and Child Health Hospital of Jiangxi Province, \\ Nanchang, China \\ ${ }^{2}$ Traditional Chinese Medicine Hospital of Taihe County, \\ Anhui Province, Taihe, China \\ Corresponding author: Z.Y. Wang \\ E-mail: zhenyingwang@yeah.net
}

Genet. Mol. Res. 14 (2): 2970-2977 (2015)

Received August 13, 2013

Accepted January 10, 2014

Published April 10, 2015

DOI http://dx.doi.org/10.4238/2015.April.10.6

\begin{abstract}
We investigated the effects of a modified Shoutaiwai recipe on integrin $\beta 3$ and leukemia-inhibitory factor (LIF) in the endometrium of controlled ovarian hyperstimulation $(\mathrm{COH})$ mice during the implantation window. Seventy non-pregnant mice were randomly divided into 3 groups: a traditional medicine (TCM) treatment group $(\mathrm{N}=30)$, an aspirin treatment $(\mathrm{N}=30)$ group, and a control group $(\mathrm{N}$ $=10$ ). After the model was successfully established, mice in the drug treatment groups and the control group were respectively treated with the modified Shoutaiwai recipe, aspirin, or $0.9 \%$ physiological saline. During the implantation window of mice, the middle segment of the mouse uterus was recovered, and integrin $\beta 3$ and LIF expressions in the endometrium were respectively detected using an immunohistological two-step method and reverse transcription-PCR. Expressions of integrin $\beta 3$ and LIF in the endometrium of mice in the TCM treatment group were
\end{abstract}


significantly increased compared to aspirin-treated and control mice, and those of aspirin-treated mice were increased compared to the control group. Our modified Shoutaiwai recipe may improve the endometrial receptivity of $\mathrm{COH}$ mice by increasing the expression of integrin $\beta 3$ and LIF in the endometrium during the implantation window.

Key words: Modified Shoutaiwai recipe; Controlled superovulation; Implantation window; Endometrial receptivity; Integrin $\beta 3$; Leukemia inhibitory factor

\section{INTRODUCTION}

With the development of assisted reproductive technology, the fertility rate of in vitro fertilization is $50-70 \%$, while the pregnancy success rate is $30-40 \%$ (Cao, 2008). Embryo nidation results in low fertilization rates and pregnancy success rates. Nidation involves a complex sequence of events and includes a variety of factors such as hormones, cytokines, adhesion molecules, and network system (Makrigiannakis and Minas, 2007), as well as endometrial receptivity, which is the receptive ability of the parent endometrium to a blastocyst. The endometrium can contain blastocysts only during an implantation window, a particular time during which positioning, adhesion, and implantation can occur. Controlled ovarian hyperstimulation $(\mathrm{COH})$ is widely used in assisted reproductive technology; however, the use of exogenous hormones at doses higher than the physiological dose reduces endometrial receptivity. The clinical use of aspirin can improve endometrial receptivity (Zhu et al., 2009), but its safety is controversial. Therefore, a safer, more effective drug should be developed. Integrins are cell adhesion molecules belonging to the integrin family that can confer a change in the endometrium from non-adhesion status to the adhesion state, and is an effective indicator for evaluating endometrial receptivity. Leukemia inhibitory factor (LIF) is a key factor affecting endometrial receptivity. LIF expression during implantation window is an important marker for determining whether the endometrium is receptive, as well as one of the promoter regulatory factors of nidation.

In this study, Alarelin, a gonadotropin-releasing hormone analog, was used to build a $\mathrm{COH}$ mouse model with reduced endometrial receptivity. Mice were treated with a modified Shoutaiwai recipe, aspirin, or $0.9 \%$ sodium chloride. In addition, integrin $\beta 3$ and LIF were used as indicators of endometrial receptivity, and PV two-step immunohistochemical staining and reverse transcription-polymerase chain reaction (RT-PCR) were used to determine the expression of integrin $\beta 3$ and LIF in uterine tissue to investigate the effects of the modified Shoutaiwai recipe on endometrial receptivity of mice during the implantation window and to provide experimental data for clinical treatment.

\section{Experimental animals and grouping}

Seventy non-pregnant female Kunming mice (10-12 weeks of age, 28.2-35.4 g) were purchased from Nanchang University Laboratory Animal Science Ministry (Jiangxi, China). The rearing conditions were as follows: free ingestion, 12-h light, ambient temperature of $18^{\circ}-22^{\circ} \mathrm{C}$, and relative humidity $60-80 \%$. The Kunming mice were randomly divided into 3 groups: traditional medicine (TCM) treatment group $(\mathrm{N}=30)$, aspirin treatment group $(\mathrm{N}=$ $30)$, and control $(0.9 \%$ sodium chloride) group $(\mathrm{N}=10)$. 


\section{Experimental drug and preparation}

For TCM, the Shoutaiwai recipe included seed, $100 \mathrm{~g}$ bark, $100 \mathrm{~g}$ Astragalus mongholicus, root, mistletoe, Chinese teasel root, macrocephala, lovage rhizome, and Codonopsis pilosula. These herbs were purchased from the Maternal and Child Health Hospital of Jiangxi Province. Its crude drug content was $2 \mathrm{~g}$, and mixture was stored in a $4^{\circ} \mathrm{C}$ refrigerator. For the Western medicine preparation, Alarelin (Shanghai GL Biochem Co., Ltd., Shanghai, China) was used. First, $10 \mathrm{mg}$ Alarelin powder was added to $20 \mathrm{~mL} 0.9 \%$ sodium chloride injection and mixed, followed by addition of $280 \mathrm{~mL} 0.9 \%$ sodium chloride.

First, $1000 \mathrm{U}$ pregnant mare serum gonadotropin (Chenguang Biotech Co., Ltd., Beijing, China) was diluted with $10 \mathrm{~mL}$ special diluent and mixed. Next, $20 \mathrm{~mL} 0.9 \%$ sodium chloride injection was adjusted to a $30 \mathrm{~mL}$ volume.

In addition, $2000 \mathrm{U}$ injection human chorionic gonadotropin (Livzon Group Pharmaceutical, Inc., Beijing, China) was added to $10 \mathrm{~mL} 0.9 \%$ sodium chloride injection and mixed. Next, $20 \mathrm{~mL} 0.9 \%$ sodium chloride injection was adjusted to a $30 \mathrm{~mL}$ volume.

Enteric-coated aspirin tablets $(600 \mathrm{mg}$; Bayer Healthcare Co., Ltd., Montville, NJ, USA) were added to $150 \mathrm{~mL} 0.5 \%$ sodium carboxymethyl cellulose solution and mixed. This formulation was stored at $4^{\circ} \mathrm{C}$.

\section{Main reagents and instruments}

The rabbit anti-mouse monoclonal antibody of integrin $\beta 3$ was purchased from $A b-$ cam, Cambridge, UK) and the immunohistochemical detection kit was purchased from Zhongshan Golden Bridge Biotechnology Co., Ltd. (China). Primer sequences were as follows: LIF mRNA: upstream 5'-CCTAACATGACAGACTTCC-3', downstream 5'-GACCACCACACTT ATGAC-3', 304 bp; $\beta$-actin: upstream 5'-TTCCAGCCTTCCTTCCTGG-3', downstream 5'-TTGCGCTCAGGAGGAGCAAT-3', 224 bp. These primers were synthetized by the Generay biological Engineering Co., Ltd. (China). TRNzol total RNA extraction reagent (DP405) and the Quant cDNA.(KR103) were purchased from Beijing, China.

\section{Administration dose}

The standard weight of a Chinese female was converted into a daily dosage for mice based on a $35 \mathrm{~g} / \mathrm{kg}$ body weight according to the body surface area calculation conversion between humans and animals (Miao, 2003). Mouse body surface area was calculated as: A (m): $\mathrm{A}_{\text {human }}=0.0061 \mathrm{x}$ height $(\mathrm{cm})+0.0128 \mathrm{x}$ body weight $(\mathrm{kg})-0.1529 ; \mathrm{A}_{\text {mice }}=\mathrm{K} \mathrm{x}$ body weight $(\mathrm{g})^{2 / 3} / 10,000$, where $\mathrm{K}$ is a constant and $\mathrm{K}_{\text {mice }}=9.1$. The dose was converted into a dosage for mice based on human dose. The following doses were used: $40 \mu \mathrm{g} / 100 \mathrm{~g}$ per $\mathrm{kg}$ x $40 \mathrm{U} / 100$ g per kg pregnant mare serum gonadotropin, $100 \mathrm{U} / 100 \mathrm{~g}$ per $\mathrm{kg}$ injection human chorionic gonadotropin, and $5 \mathrm{mg} / 100 \mathrm{~g}$ per $\mathrm{kg}$ aspirin enteric-coated tablets.

\section{Establishment of animal model and evaluation criteria}

Mice in TCM treatment group, aspirin treatment group, and control group were injected intraperitoneally with Alarelin, once a day, at 9:00 am in the third day of the estrous cycle. After 
9 days, $0.4 \mathrm{~mL}$ pregnant mare serum gonadotropin was added and $0.4 \mathrm{~mL} \mathrm{hCG}$ was injected again $48 \mathrm{~h}$ after injection. After superovulation drug administration, vaginal secretions of mice were increased significantly, and a large number of non-nuclear keratinocyte secretions were observed under light microscopy, confirming that the animal model was successfully established.

\section{Administration method}

Mice in the TCM treatment group were administered $0.4 \mathrm{~mL}$ modified Shoutaiwai recipe once a day. Mice in the aspirin treatment group were administered $0.4 \mathrm{~mL}$ aspirin sodium carboxymethyl cellulose solution by gavage. Mice in the control group were administered 0.4 $\mathrm{mL} 0.9 \%$ sodium chloride injection by gavage once every 11 days.

\section{Specimen collection}

Forty-eight hours after injecting hCG during the implantation window, mice in each group were euthanized, the uterus was removed by cesarean section, and the surrounding mucosa was removed. The middle segment of the uterus was fixed with $4 \%$ paraformaldehyde solution and RNAstore sample preservation solution.

\section{Expression of integrin $\beta 3$ determined by immunohistochemical PV two-step method}

After fixation, dehydration, and other treatment for uterine tissue, staining steps were carried out according to the immunohistochemical detection kit instructions. Rabbit antimouse integrin- $\beta 3$ monoclonal antibody $(1: 100)$ was used for detection. Brown particles were considered as positive staining.

\section{Result criteria}

Five fields for every slice were chosen under optical microscopy (400) and 100 cells were counted per field. Scoring was evaluated as follows: negative $(-),<5 \%$ positive cell count; weakly positive $(+), 5-15 \%$ positive cell count; positive $(++), 16-50 \%$ positive cell count; strongly positive $(+++),>50 \%$ positive cells. For staining intensity, scoring was as follows: negative (-), no stained cells; + , light yellow; ++ , brownish yellow; +++ , brown. Expression intensity was evaluated based on positive cells and staining by a pathologist: -, 0 score; +, 1 score; ++, 2 score; +++, 3 score (Xing et al., 2001).

\section{RT-PCR}

Total RNA in mouse endometria was extracted using Trizol reagent and stored at $-80^{\circ} \mathrm{C}$. Next, $20 \mu \mathrm{L}$ reverse transcriptase reaction was incubated in an electric thermostat water bath at $37^{\circ} \mathrm{C}$ for 60 min to obtain a cDNA product. PCR amplification conditions were as follows: initial denaturation at $94^{\circ} \mathrm{C}, 3 \mathrm{~min} ; 94^{\circ} \mathrm{C}, 30 \mathrm{~s} ; 63.8^{\circ} \mathrm{C}, 30 \mathrm{~s} ; 72^{\circ} \mathrm{C}, 60 \mathrm{~s}$; total of 30 cycles, followed by extension at $72^{\circ} \mathrm{C}$ for $10 \mathrm{~min}$. The amplification product was evaluated by using $1.5 \%$ agarose gel electrophoresis and observed under an ultraviolet transilluminator. PCR amplification products were analyzed by using the Image J software (NIH, Bethesda, 
MD, USA), and the ratio of the OD value of LIF mRNA to OD values of $\beta$-actin amplification bands was considered the relative LIF mRNA expression.

\section{Statistical analysis}

The SPSS 18.0 software (SPSS, Inc., Chicago, IL, USA) was used for statistical analysis. The $\chi^{2}$ test was used for enumeration and non-ranked data. Non-parametric Ridit analysis was used for enumeration and ranked data. One-way analysis of variance was used for measurement data. $\mathrm{P}<0.05$ was considered to be statistically significant.

\section{RESULTS}

\section{Expression of integrin $\beta 3$}

Integrin $\beta 3$ was primarily expressed in the cytoplasm of endometrial glandular epithelial cells during the implantation window. Positive and strong positive expression of integrin $\beta 3$ in the TCM treatment group was 36.7 and $46.7 \%$, respectively, and staining was brown. Positive and strong positive expression of integrin $\beta 3$ in the aspirin treatment group was 43.3 and $23.3 \%$, respectively, and main staining was brownish and yellow. Positive expression of integrin $\beta 3$ in the control group was only $20 \%$, and no strong positive expression and pale yellow staining or no staining was observed. Table 1 and Figure $1 \mathrm{~A}-\mathrm{C}$ show these results. Ridit analysis showed that the R value for the TCM treatment group was 0.479 , for the aspirin treatment group it was 0.214 , and for the control group, 0.500 . The expression of integrin $\beta 3$ in the TCM treatment group was significantly increased compared to the aspirin group $(\mathrm{P}=0.034, \mathrm{P}$ $<0.01)$. Expression of integrin $\beta 3$ in the aspirin group was significantly increased compared to that in control mice $(\mathrm{P}=0.005$; Table 1$)$.

Table 1. Comparison of protein expression level of integrin $\beta 3$ in endometrium on each group (\%).

\begin{tabular}{lccccc}
\hline Group & Mice (N) & \multicolumn{4}{c}{ Staining degree } \\
\cline { 3 - 5 } & & - & + & ++ & +++ \\
\hline TCM & 30 & $1(3.3 \%)$ & $4(13.3 \%)$ & $11(36.7 \%)$ & $14(46.7 \%)^{*}$ \\
Aspirin & 30 & $3(10 \%)$ & $7(23.3 \%)$ & $13(43.3 \%)$ & $7(23.3 \%)^{\triangle}$ \\
Control & 10 & $4(40 \%)$ & $4(40 \%)$ & $2(20 \%)$ & $0(0)$ \\
\hline
\end{tabular}

$*$ Traditional medicine group (TCM) group $v s$ aspirin group; ${ }^{\triangle}$ aspirin group $v s$ control group; TCM group $v s$ control group: $\mathrm{P}<0.01$.
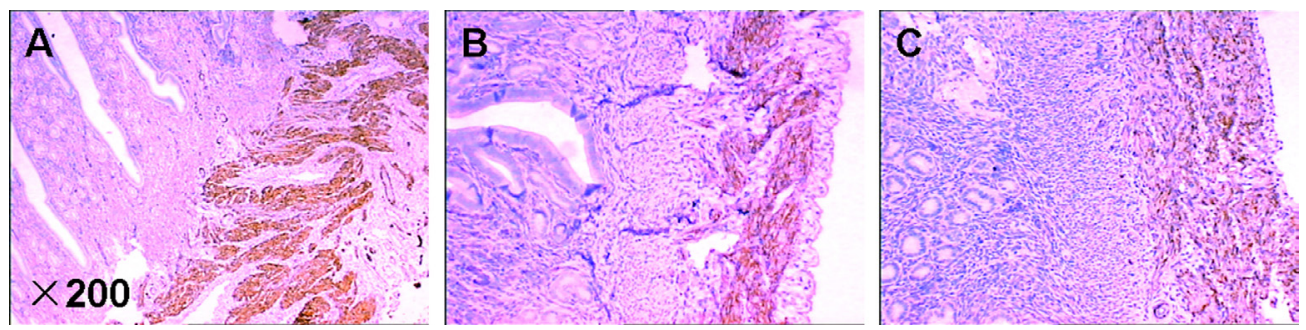

Figure 1. Expressions of integrin $\beta 3$ in endometrium of mice on each group (immunohischemical PV two-step staining). A. Traditional medicine group. B. Aspirin group. C. Control group. 


\section{Ratio of LIF mRNA/ $\beta$-actin in the endometrium in each group}

Table 2 shows the ratios of LIF mRNA to actin in the endometrium in the three groups. LIF mRNA expression in TCM and aspirin treatment groups was significantly increased compared to that of the control group $(\mathrm{P}<0.01)$. LIF mRNA expression in the TCM treatment group was significantly increased compared to that in the aspirin group $(\mathrm{P}=0.02$; Figure 2$)$.

Table 2. Ratio of LIF mRNA to $\beta$-actin in endometria of $\mathrm{COH}$ mice during implantation window.

\begin{tabular}{lccc}
\hline Group & Mice $(\mathrm{N})$ & Means \pm SD & $\mathrm{P}$ \\
\hline TCM & 30 & $0.9835 \pm 0.0059$ & $0.02^{\mathrm{a}}$ \\
Aspirin & 30 & $0.9793 \pm 0.0061$ & $<0.01^{\mathrm{b}}$ \\
Control & 10 & $0.9670 \pm 0.0103$ & \\
\hline
\end{tabular}

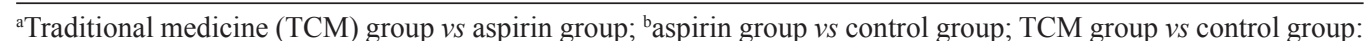
$\mathrm{P}<0.01$. LIF $=$ leukemia-inhibitory factor; $\mathrm{COH}=$ controled ovarian hyperstimulation.

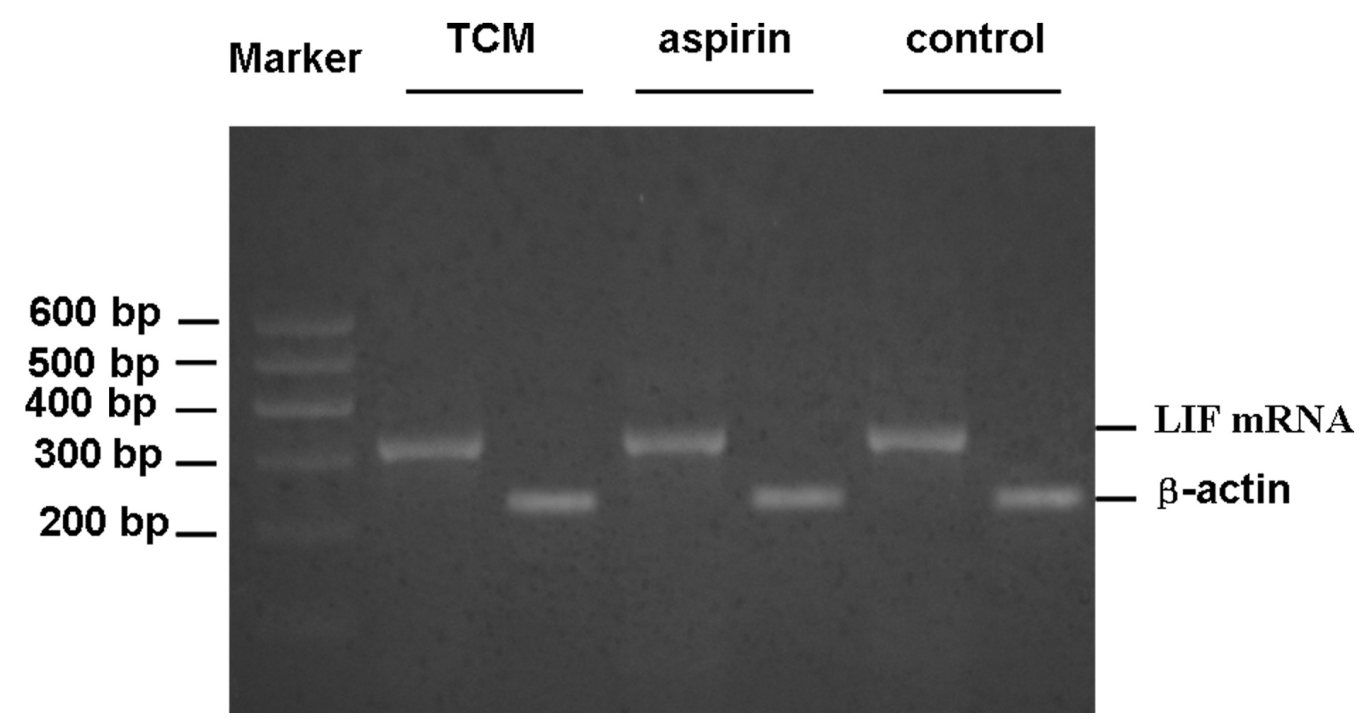

Figure 2. mRNA expressions of LIF and $\beta$-actin in endometria of $\mathrm{COH}$ mice during implantation window. For abbreviations, see legend to Table 2 .

\section{DISCUSSION}

With the sophisticated development of assisted reproductive technology, $\mathrm{COH}$ has been widely used clinically. At higher than physiological doses, the exogenous hormone can result in morphological changes in the endometrium, abnormal expression of markers, and reduced endometrial receptivity. Gao and Zhang (2005) found that during the down-regulation phase of superovulation, gonadotropin-releasing hormone analogs could decrease endometrial integrin expression during nidation, resulting in reduced endometrial receptivity. Integrin is an important cell adhesion molecule that can recognize extracellular matrix pro- 
teins based on the integrin arginine-glycine-aspartic acid sequence, which mediates cell adhesion to the extracellular matrix, promotes production of angiogenesis factors in conjunction with a variety of signals, mediates intracellular and extracellular transduction, and increases the blood supply to the endometrium, thereby improving endometrial receptivity (Chan et al., 2006; Zhang et al., 2009).

Integrin 3 is secreted in the endometrial glandular epithelium and luminal epithelium 19 days after the normal menstrual cycle, and is involved in the adhesion of blastocyst implantation. A previous study confirmed that integrin 3 was not or was weakly expressed in infertile women during the implantation window (Boroujerdnia and Nikbakht, 2008).

LIF, a glycoprotein secreted by natural killer cells, is a member of the interleukin-6 family of cytokines that can regulate human reproduction (Kondera-Anasz et al., 2004). Acosta et al. (2000) found that the mRNA of LIF was expressed during the menstrual cycle, and, in particular, was highly expressed during the late phase and mid-phase of the secretory endometrium of the menstrual cycle and early pregnancy. Aghajanova et al. (2003) confirmed that initiation of human embryonic development depends on molecular changes and cell morphology changes of LIF and its receptor, as well as pinocytosis. A sudden, strong expression occurs during the endometrial implantation window, and LIF expression and pinocytosis are synchronized with disappearance, suggesting that LIF plays an important role in the embryonic development, implantation, and pregnancy maintenance phases.

Clinically, aspirin can improve endometrial receptivity to increase the success rate of pregnancy after embryo transfer. A previous study (Li and Deng, 2006) confirmed that aspirin can inhibit the local adverse effects of inflammatory mediators on the systemic and reproductive system through immunomodulatory effects, thereby inhibiting uterine vasoconstriction and ovarian and platelet aggregation to improve the blood supply to the endometrium and improve endometrial receptivity. The results suggest that aspirin can improve endometrial receptivity in $\mathrm{COH}$ mice by increasing the expression of endometrial integrin 3 .

A large number of studies have been conducted to examine the side effects of aspirin. Carnovale et al. (2001) reported that aspirin could inhibit prostaglandin in a dose-dependent manner, which is the main prostaglandin involved in cultivation and decidualization, cytokine production, and ovulation. In addition, aspirin induces embryo teratogenicity (Streck et al., 2003), drawing attention to its toxicity. Kozer et al. (2002) conducted a meta-analysis showing that aspirin exposure during the first trimester may be associated with an increased risk of gastroschisis.

The Shoutaiwai recipe has the effect of invigorating the kidney. Recent pharmacological studies confirmed that its main effect is in preventing miscarriage and promoting ovarian development. Liu and You (2004) investigated its molecular mechanisms and showed that it not only acts on receptors, but also regulates the balance of sex hormones. Zhang et al. (2008) evaluated the effects on the mouse endometrium, Bushenyiqihuoxue of Chinese medicine showed that the treatment could not only maintain endometrial cell decidualization and embryo implantation, but could also improve local cyclooxygenase- 2 expression, facilitating embryo implantation. Zhou et al. (2009) confirmed that Bushen zhuyun recipe could increase the expression level of integrin 3, improving the luteal phase defect state and endometrial receptivity. Experimental Shoutai pills, as part of the Chinese medicine Bushenjianpihuoxue method, affected endometrial integrin 3 expression and improved $\mathrm{COH}$ of mice during the implantation window for endometrial receptivity.

We confirmed that the expressions of integrin 3 and LIF in the TCM and aspirin groups 
were significantly improved compared to the control group $(\mathrm{P}<0.01)$, suggesting that both treatments can improve the hyperstimulation of endometrial integrin 3 and LIF expression in mice. Integrin 3 expression in the TCM group was significantly better than that in the aspirin group (P 0.05), indicating that the Shoutai pill can improve endometrial hyperstimulation in mice and that integrin $\beta 3$ and LIF expression is improved compared to levels following aspirin treatment. The Shoutai pill can improve superovulation of mouse endometrium, integrin $\beta 3$, LIF expressions, and hyperstimulation to increase endometrial receptivity. Clinical applications of the superovulation treatment and effects on improved human endometrial receptivity require further analysis.

\section{REFERENCES}

Acosta AA, Elberger L, Borghi M, Calamera JC, et al. (2000). Endometrial dating and determination of the window of implantation in healthy fertile women. Fertil. Steril. 73: 788-798.

Aghajanova L, Stavreus-Evers A, Nikas Y, Hovatta O, et al. (2003). Coexpression of pinopodes and leukemia inhibitory factor, as well as its receptor, in human endometrium. Fertil. Steril. 79 (Suppl 1): 808-814.

Boroujerdnia MG and Nikbakht R (2008). Beta3 integrin expression within uterine endometrium and its relationship with unexplained infertility. Pak. J. Biol. Sci. 11: 2495-2499.

Cao ZY (2008). Obstetrics and Gynecology. People's Health Publishing House, Beijing.

Carnovale DE, Fukuda A, Underhill DC, Laffan JJ, et al. (2001). Aspirin dose dependently inhibits the interleukin-1 betastimulated increase in inducible nitric oxide synthase, nitric oxide, and prostaglandin $\mathrm{E}(2)$ production in rat ovarian dispersates cultured in vitro. Fertil. Steril. 75: 778-784.

Chan PC, Chen SY, Chen CH and Chen HC (2006). Crosstalk between hepatocyte growth factor and integrin signaling pathways. J. Biomed. Sci. 13: 215-223.

Gao J and Zhang HW (2005). Effect of controlled ovarian hyperstimulation assisted by GnRHa on the thickness of implantation endomentrium and the expression of integrins in mouse. Prog. Obstet. Gynecol. 14: 135-137.

Kondera-Anasz Z, Sikora J and Mielczarek-Palacz A (2004). Leukemia inhibitory factor: an important regulator of endometrial function. Am. J. Reprod. Immunol. 52: 97-105.

Kozer E, Nikfar S, Costei A, Boskovic R, et al. (2002). Aspirin consumption during the first trimester of pregnancy and congenital anomalies: a meta-analysis. Am. J. Obstet. Gynecol. 187: 1623-1630.

Li R and Deng QJ (2006). The using of anti-coagulation drugs in the reproductive fields. Chin. J. Pract. Gynecol. Obstet. 22: $169-170$.

Liu HP and You ZL (2004). The effect of Shoutai pill on SOCS1 expression in decidua of the mice with spontaneous abortion. Guiding J. TCM 14: 6-8.

Makrigiannakis A and Minas V (2007). Mechanisms of implantation. Reprod. Biomed. Med. Online 14: 102-109.

Miao MS (2003). Experimental Animals and Animal Experimental Techniques. Traditional Chinese Medicine Press, Beijing.

Streck RD, Kumpf SW, Ozolins TR and Stedman DB (2003). Rat embryos express transcripts for cyclooxygenase-1 and carbonic anhydrase-4, but not for cyclooxygenase-2, during organogenesis. Birth Defects Res. B Dev. Reprod. Toxicol. 68: 57-69.

Xing CP, Liu B and Dong L (2001). The judgment method of immunohistochemical labeling result. Chin. J. Pathol. 30: 318.

Zhang MM, Huang YQ and Cheng LL (2008). Effect of Bushenantai recipe on the expression of endometrial HB-EGF and EGFR in mice with embryonic implantation dysfunction. J. Huazhong Univ. Sci. Technol. 37: 85-88.

Zhang Y, Wang JH, Liu X and Wei LQ (2009). Arg-Gly-Asp-motif toxins using integrin as a target: research advances. J. Int. Pharm. Res. 36: 406-411.

Zhou HF, Li AP and Tan Y (2009). Effects of Bushen Zhuyun recipe on protein expression of estrogen receptor, progesterone receptor and integrin alpha5 and beta3 in endometrium of rats at the implantation stage. Zhongguo Zhong Xi Yi Jie He Za Zhi 29: 628-631.

Zhu H, Wang F and Li B (2009). The progress of Chinese and Western medicine on endometrial receptivity. J. Int. Obstet. Gynecol. 36: 360-363. 